\title{
Normality of Meromorphic Functions and Uniformly Discrete Exceptional Sets
}

\author{
Jianming Chang
}

Received: 11 May 2012 / Revised: 10 September 2012 / Accepted: 8 December 2012 /

Published online: 23 January 2013

(C) The Author(s) 2013. This article is published with open access at Springerlink.com

\begin{abstract}
Let $k \in \mathbb{N}$ and $h(\not \equiv 0)$ be a function holomorphic on $D$. Let $\mathcal{F}$ be a family of meromorphic functions in $D$, all of whose zeros have multiplicity at least $k+3$. Suppose that the sets $\left\{E_{f}\right\}_{f \in \mathcal{F}}$ are locally uniformly discrete in $D$, where $E_{f}=\{z \in D: f(z)=0\} \cup\left\{z \in D: f^{(k)}(z)=h(z)\right\}$. Suppose additionally that at the common zeros of $f \in \mathcal{F}$ and $h$, the multiplicities $m_{f}$ for $f$ and $m_{h}$ for $h$ satisfy $m_{f} \geq m_{h}+k+1$ for $k>1$ and $m_{f} \geq 2 m_{h}+3$ for $k=1$. Then, $\mathcal{F}$ is normal in $D$. The number $k+3$ can be replaced by $k+2$ if the set $E_{f}$ is independent of $f$, or in other words, for each pair of functions $f$ and $g$ in $\mathcal{F}, f$ and $g$ share the value 0 , and $f^{(k)}$ and $g^{(k)}$ share the function $h$. Examples are also given to show that the conditions are necessary and sharp.
\end{abstract}

Keywords Meromorphic function · Normal family · Shared values ·

Locally uniformly discrete sets

Mathematics Subject Classification (2000) Primary 30D45

\section{Introduction and Main Results}

A family $\mathcal{F}$ of meromorphic functions defined in a plane domain $D \subset \mathbb{C}$ is said to be normal in $D$, if each sequence $\left\{f_{n}\right\} \subset \mathcal{F}$ contains a subsequence which converges spherically locally uniformly in $D$ to a meromorphic function or $\infty$. See $[6,11,15]$.

Communicated by Lawrence Zalcman.

Research supported by NNSF of China, Grant No. 11171045.

J. Chang $(\varangle)$

Department of Mathematics, Changshu Institute of Technology,

Changshu, Jiangsu 215500, People's Republic of China

e-mail: jmchang@cslg.edu.cn 
The Gu's normality criterion [5] which was conjectured by Hayman [6] says that a family $\mathcal{F}$ of functions meromorphic on $D$ is normal if $f \neq 0$ and $f^{(k)} \neq 1$ for each $f \in \mathcal{F}$. The following generalization of Gu's theorem was proved by Yang [14].

Theorem 1 Let $\mathcal{F}$ be a family of meromorphic functions on $D, k \in \mathbb{N}$ and $h(\not \equiv 0)$ be a holomorphic function on $D$. If for every $f \in \mathcal{F}, f \neq 0$ and $f^{(k)} \neq h$ on $D$, then $\mathcal{F}$ is normal on $D$.

In recent years, following Schwick [12], many normality criteria concerning shared values or functions have been proved. We say that two functions $f$ and $g$ share a value or a function $\phi$ if the two equations $f(z)=\phi(z)$ and $g(z)=\phi(z)$ have the same solutions (ignoring multiplicity). Here, we want to generalize the following result of Fang and Zalcman [4] by replacing the constant 1 by a function.

Theorem 2 Let $k$ be a positive integer and let $\mathcal{F}$ a family of meromorphic functions on $D$, all of whose zeros have multiplicity at least $k+2$, such that for each pair of functions $f$ and $g$ in $\mathcal{F}, f$ and $g$ share the value 0 , and $f^{(k)}$ and $g^{(k)}$ share the value 1 . Then, the family $\mathcal{F}$ is normal.

In general, the constant 1 cannot be replaced by a function. For example, the family $\left\{f_{n}\right\}$, where $f_{n}(z)=n z^{k+2}$, is not normal at 0 . However, each pair of functions $f_{n}$ and $f_{m}$ share the value 0 , and $f_{n}^{(k)}$ and $f_{m}^{(k)}$ share the function $z^{2}$.

So we need some additional conditions. We prove the following generalization of Theorem 2.

Theorem 3 Let $k \in \mathbb{N}$ and $h(\not \equiv 0)$ be a function holomorphic on $D$. Let $\mathcal{F}$ be a family of meromorphic functions in $D$, all of whose zeros have multiplicity at least $k+2$, such that for each pair of functions $f$ and $g$ in $\mathcal{F}, f$ and $g$ share the value 0 , and $f^{(k)}$ and $g^{(k)}$ share the function $h$. Suppose additionally that at each common zero of $f$ and $h$ for every $f \in \mathcal{F}$, the multiplicities $m_{f}$ for $f$ and $m_{h}$ for $h$ satisfy $m_{f} \geq m_{h}+k+1$ for $k>1$ and $m_{f} \geq 2 m_{h}+3$ for $k=1$. Then, $\mathcal{F}$ is normal in $D$.

The above example shows that the assumption $m_{f} \geq m_{h}+k+1$ is necessary. The condition $m_{f} \geq 2 m_{h}+3$ for $k=1$ is also necessary and sharp as showed by the following example.

Example 1 Let $\alpha$ be a positive integer and $h(z)=z^{\alpha}$. Let for $n \in \mathbb{N}$,

$$
f_{n}(z)=\frac{z^{2 \alpha+2}}{(\alpha+1)\left[z^{\alpha+1}-1 / n\right]}
$$

Then,

$$
f_{n}^{\prime}(z)-h(z)=\frac{z^{3 \alpha+2}-\frac{2}{n} z^{2 \alpha+1}}{\left(z^{\alpha+1}-\frac{1}{n}\right)^{2}}-z^{\alpha}=-\frac{\frac{1}{n^{2}} z^{\alpha}}{\left(z^{\alpha+1}-\frac{1}{n}\right)^{2}} .
$$

Thus, each pair of functions $f_{n}$ and $f_{m}$ share the value 0 , and $f_{n}^{\prime}$ and $f_{m}^{\prime}$ share the function $h(z)$. However, the family $\left\{f_{n}\right\}$ is not normal at 0 . 
Let us look at some more aspects. By fixing a function $f_{0} \in \mathcal{F}$ and letting $E=$ $\left\{z \in D: f_{0}(z)=0\right\} \cup\left\{z \in D: f_{0}^{(k)}(z)=h(z)\right\}$, the shared condition of Theorem 3 is equivalent to saying that there exists a fixed set $E \subset D$, which is independent of $f \in \mathcal{F}$, such that $f \neq 0$ and $f^{(k)} \neq h$ on $D \backslash E$ for every $f \in \mathcal{F}$. There are two cases for the set $E$. One which is trivial is that $E=D$. Then, every $f \in \mathcal{F}$ satisfies $f \equiv 0$ or $f^{(k)} \equiv h$, and hence the normality of $\mathcal{F}$ can be easily dealt with. The non-trivial case is that the exceptional set $E$ is (locally) discrete in $D$. We focus on the non-trivial case and consider here that the exceptional set $E$ is dependent on $f$. To state our result, we require the following definition.

Definition 1 The sets $\left\{E_{\lambda}\right\}_{\lambda \in \Lambda}$ are said to be locally uniformly discrete in $D$, if for each point $z_{0} \in D$, there exists $\delta>0$ such that every $E_{\lambda}$ has at most one point lying in the disk $\Delta\left(z_{0}, \delta\right)=\left\{z:\left|z-z_{0}\right|<\delta\right\}$.

For example, the sets $\left\{E_{n}\right\}_{n \in \mathbb{N}}$, where $E_{n}=\left\{\frac{m-1}{m}+\frac{1}{n}: m \in \mathbb{N}\right\}$, are locally uniformly discrete in the unit disk $\Delta(0,1)$, but not in the domains that contain the point 1.

Theorem 4 Let $k \in \mathbb{N}$ and $h(\not \equiv 0)$ be a function holomorphic on $D$. Let $\mathcal{F}$ be a family of meromorphic functions in $D$, all of whose zeros have multiplicity at least $k+2$ or $k+3$ and $h$ has simple zeros. Suppose that the sets $\left\{E_{f}\right\}_{f \in \mathcal{F}}$, where $E_{f}=\{z \in D$ : $f(z)=0\} \cup\left\{z \in D: f^{(k)}(z)=h(z)\right\}$, are locally uniformly discrete in D. Suppose additionally that at the common zeros of $f \in \mathcal{F}$ and $h$, the multiplicities $m_{f}$ for $f$ and $m_{h}$ for $h$ satisfy $m_{f} \geq m_{h}+k+1$ for $k>1$ and $m_{f} \geq 2 m_{h}+3$ for $k=1$. Then, $\mathcal{F}$ is normal in $D$.

The following example shows that it is necessary (and sharp) to assume that the multiplicity of zeros of $f \in \mathcal{F}$ is at least $k+3$ when $h$ has simple zeros.

Example 2 Let $h(z)=z$ and

$$
f_{n}(z)=\frac{(z-1 / n)^{k+2}}{(k+1) ![z-(k+2) / n]}, \quad n \in \mathbb{N} .
$$

Then, $E_{f_{n}}=\{1 / n\}$, so that $\left\{E_{f_{n}}\right\}$ are locally uniformly discrete in $\mathbb{C}$. In fact, we have

$$
\begin{aligned}
f_{n}(z)= & \frac{\left[\left(z-\frac{k+2}{n}\right)+\frac{k+1}{n}\right]^{k+2}}{(k+1) !\left(z-\frac{k+2}{n}\right)}=\frac{1}{(k+1) !}\left[\left(z-\frac{k+2}{n}\right)^{k+1}\right. \\
& \left.+\frac{(k+1)(k+2)}{n}\left(z-\frac{k+2}{n}\right)^{k}+P(z)+\frac{\left(\frac{k+1}{n}\right)^{k+2}}{\left(z-\frac{k+2}{n}\right)}\right],
\end{aligned}
$$

where $P$ is a polynomial of degree $<k$, so that

$$
f_{n}^{(k)}(z)=z+\frac{(-1)^{k}\left(\frac{k+1}{n}\right)^{k+2}}{(k+1)\left(z-\frac{k+2}{n}\right)^{k+1}} \neq h(z) .
$$


However, $\left\{f_{n}\right\}$ is not normal at 0 , as $f_{n}(1 / n)=0$ while $f_{n}((k+2) / n)=\infty$. Throughout in this paper, we denote by $\mathbb{C}$ the complex plane, by $\mathbb{C}^{*}$ the punctured complex plane $\mathbb{C} \backslash\{0\}$, by $\Delta\left(z_{0}, r\right)$ the open disk $\left\{z:\left|z-z_{0}\right|<r\right\}$, and by $\Delta^{\circ}\left(z_{0}, r\right)$ the punctured disk $\Delta\left(z_{0}, r\right) \backslash\left\{z_{0}\right\}=\left\{z: 0<\left|z-z_{0}\right|<r\right\}$, where $z_{0} \in \mathbb{C}$ and $r>0$.

\section{Auxiliary Results}

To prove our results, we require some preliminary results.

Lemma 5 [3] Let $\mathcal{F}$ be a family of meromorphic functions in a domain $D$, all of whose zeros have multiplicity at least $k$. Then, if $\mathcal{F}$ is not normal at $z_{0}$, there exist, for each $-1<\alpha<k$, points $z_{n} \in D$ with $z_{n} \rightarrow z_{0}$, functions $f_{n} \in \mathcal{F}$ and positive numbers $\rho_{n} \rightarrow 0$ such that $g_{n}(\zeta):=\rho_{n}^{-\alpha} f_{n}\left(z_{n}+\rho_{n} \zeta\right)$ converges locally uniformly with respect to the spherical metric in $\mathbb{C}$ to a non-constant meromorphic function $g$ of finite order, all of whose zeros have multiplicity at least $k$.

The original form $(\alpha=0)$ of this rescaling lemma is due to Zalcman [16], while the case $-1<\alpha<1$ was proved by Pang [8,9]. The present form is due to Chen and $\mathrm{Gu}$ [3]. This lemma also holds for $\alpha=k$ [10] under an additional condition.

Lemma 6 [6,7] Let $k$ be a positive integer, $f$ be a transcendental meromorphic function and $P(\equiv 0)$ be a polynomial. Then, either $f$ or $f^{(k)}-P$ has infinitely many zeros. If $f$ is a non-constant rational function, then either $f$ or $f^{(k)}-1$ has at least one zero.

Lemma 7 [13] Let $k$ be a positive integer and let $f$ be a non-constant rational function such that $f^{(k)} \neq 1$ on $\mathbb{C}$. Then, either $f$ is a polynomial of degree at most $k$ or $f(z)=z^{k} / k !+P_{k}(z)+a(z-b)^{-n}$, where $a(\neq 0), b \in \mathbb{C}$ and $n \in \mathbb{N}$ are constants, and $P_{k}$ is a polynomial of degree less than $k$. Furthermore, $f$ has a zero whose multiplicity is at most $k+1$.

Lemma 8 [2, Lemma 4] Let $k$ be a positive integer and $f$ be a non-constant rational function. If $f(z) \neq 0$ for $z \in \mathbb{C}$, then $f^{(k)}-1$ has at least $k+1$ distinct zeros on $\mathbb{C}$.

Lemma 9 Let $k$ be a positive integer and $f$ be a non-constant rational function. If $f(z)\left[f^{(k)}(z)-1\right] \neq 0$ for $z \in \mathbb{C} \backslash\left\{z_{0}\right\}$, where $z_{0} \in \mathbb{C}$, then $z_{0}$ is a zero of $f$ with multiplicity at most $k+1$.

Proof We claim that $f\left(z_{0}\right)=0$. For otherwise, we would have $f(z) \neq 0$ for $z \in \mathbb{C}$ by the condition, and hence, by Lemma $8, f^{(k)}-1$ has at least $k+1 \geq 2$ distinct zeros, which contradicts that $f^{(k)}(z) \neq 1$ for $z \neq z_{0}$.

We now assume that the zero $z_{0}$ of $f$ has multiplicity at least $k+2$. Then, $f^{(k)}\left(z_{0}\right)=$ 0 . Hence by the condition, we have $f(z) \neq 0$ for $z \in \mathbb{C} \backslash\left\{z_{0}\right\}$, and $f^{(k)}(z) \neq 1$ for $z \in \mathbb{C}$. Thus by Lemma 7, $f$ has a zero whose multiplicity is at most $k+1$. Since $f(z) \neq 0$ for $z \in \mathbb{C} \backslash\left\{z_{0}\right\}$, this zero coincides with $z_{0}$, which contradicts the assumption that the zero $z_{0}$ of $f$ has multiplicity at least $k+2$.

Thus, $z_{0}$ is a zero of $f$ with multiplicity at most $k+1$. 
Lemma 10 Let $k, m$ be positive integers and $f$ be a non-constant rational function. If $f^{(k)}(z) \neq z^{m}$ for $z \in \mathbb{C}$ and if $f(z) \neq 0$ for $z \neq z_{0}$, where $z_{0} \in \mathbb{C}$, then $m=1$, $z_{0} \neq 0$ and either $f(z)=\left(z-z_{0}\right)^{k+1} /(k+1)$ ! or

$$
f(z)=\frac{1}{(k+1) !} \cdot \frac{\left(z-z_{0}\right)^{k+2}}{z-(k+2) z_{0}}
$$

Proof Consider first the case that $f$ is a non-constant polynomial. Then by $f(z) \neq 0$ for $z \neq z_{0}, f(z)=C_{1}\left(z-z_{0}\right)^{l}$ for some constant $C_{1} \neq 0$ and $l \in \mathbb{N}$; and by $f^{(k)}(z) \neq$ $z^{m}, f^{(k)}(z)=z^{m}+C_{2}$ for some constant $C_{2} \neq 0$. Thus, $C_{1}\left[\left(z-z_{0}\right)^{l}\right]^{(k)}=z^{m}+C_{2}$. It can be easily seen that $z_{0} \neq 0, m=1, l=k+1$ and $C_{1}=1 /(k+1)$ !. Hence $f(z)=\left(z-z_{0}\right)^{k+1} /(k+1) !$.

Now we assume that $f$ is a non-polynomial rational function. By $f^{(k)}(z) \neq z^{m}$ for $z \in \mathbb{C}$, we have

$$
\left[f(z)-\frac{m !}{(m+k) !} z^{m+k}+\frac{1}{k !} z^{k}\right]^{(k)}=f^{(k)}(z)-z^{m}+1 \neq 1 .
$$

Thus by Lemma 7,

$$
f(z)=\frac{m !}{(m+k) !} z^{m+k}+P_{k}(z)+\frac{a}{(z-b)^{n}},
$$

where $P_{k}, a, b, n$ are stated as in Lemma 7. Since $f(z) \neq 0$ for $z \neq z_{0}$, we also get $f(z)=C\left(z-z_{0}\right)^{l}(z-b)^{-n}$, for some constant $C \neq 0$ and integer $l \geq 0$. This, combined with (1), yields that

$$
\left[\frac{m !}{(m+k) !} z^{m+k}+P_{k}(z)\right](z-b)^{n}=C\left(z-z_{0}\right)^{l}-a .
$$

Comparing the degree and the coefficient of the leading term of (2) yields that $l=$ $m+k+n$ and $C=\frac{m !}{(m+k) !}$. Further, since each zero of the right hand side of (2) is simple, we see that $n=1$. Thus, $l=m+k+1$ and we can deduce from (2) that

$$
\left[\frac{m !}{(m+k) !} z^{m+k}+P_{k}(z)\right](z-b)=\frac{m !}{(m+k) !}\left(z-z_{0}\right)^{m+k+1}-a .
$$

Now by comparing the coefficients of the term $z^{m+k}$, we get $b=(m+k+1) z_{0}$.

We claim that $z_{0} \neq 0$. In fact, if $z_{0}=0$, then $b=0$, and hence $a=0$ by taking $z=0$ in (3). This is a contradiction.

Next by comparing the coefficients of the term $z^{m+k-1}$, we see that $m=1$. Thus, $f$ has the second desired form.

Lemma 11 Let $k, m$ be positive integers and $f$ be a rational function. If $f(z) \neq 0$ for $z \in \mathbb{C}$, and $f^{(k)}(z) \neq z^{m}$ for $z \neq z_{0}$, where $z_{0} \in \mathbb{C}$, then $f$ is a constant. 
Proof If $f$ is a polynomial, then by $f(z) \neq 0, f$ must be constant. Now suppose that $f$ is a non-polynomial rational function. Then by Lemma $10, f^{(k)}(z)-z^{m}$ must have at least one zero. Hence by the condition, $f^{(k)}\left(z_{0}\right)=z_{0}^{m}$. Thus by $f(z) \neq 0$ for $z \in \mathbb{C}$ and $f^{(k)}(z) \neq z^{m}$ for $z \neq z_{0}$, we can write

$$
f(z)=C_{1} \prod_{i=1}^{n}\left(z-z_{i}\right)^{-p_{i}}, f^{(k)}(z)=z^{m}+C_{2}\left(z-z_{0}\right)^{l} \prod_{i=1}^{n}\left(z-z_{i}\right)^{-p_{i}-k},
$$

where $C_{1}, C_{2}$ are non-zero constants, $l, n, p_{i}$ are positive integers, and $z_{i}, 0 \leq i \leq n$ are distinct complex numbers. By the expression of $f$ in (4), we have

$$
f^{(k)}(z)=P(z) \prod_{i=1}^{n}\left(z-z_{i}\right)^{-p_{i}-k}
$$

where $P$ is polynomial of degree $(n-1) k$. Thus by the two expressions of $f^{(k)}$ in (4) and (5),

$$
z^{m} \prod_{i=1}^{n}\left(z-z_{i}\right)^{p_{i}+k}+C_{2}\left(z-z_{0}\right)^{l}=P(z) .
$$

Since

$$
\operatorname{deg}(P)=(n-1) k<m+\sum_{i=1}^{n}\left(p_{i}+k\right)=\operatorname{deg}\left(z^{m} \prod_{i=1}^{n}\left(z-z_{i}\right)^{p_{i}+k}\right),
$$

by (6), we have

$$
l=m+\sum_{i=1}^{n}\left(p_{i}+k\right)=m+k n+\sum_{i=1}^{n} p_{i}
$$

and $C_{2}=-1$. By letting $z=1 / t$ in (6), we get

$$
\prod_{i=1}^{n}\left(1-z_{i} t\right)^{p_{i}+k}=\left(1-z_{0} t\right)^{l}+t^{l} P(1 / t)=\left(1-z_{0} t\right)^{l}\left[1+O\left(t^{m+k+\sum_{i=1}^{n} p_{i}}\right)\right]
$$

as $t$ goes to 0 . Thus by taking the logarithmic derivatives,

$$
\sum_{i=1}^{n} \frac{\left(p_{i}+k\right) z_{i}}{1-z_{i} t}-\frac{l z_{0}}{1-z_{0} t}=O\left(t^{m+k-1+\sum_{i=1}^{n} p_{i}}\right)
$$

It follows that 


$$
\sum_{i=1}^{n}\left(p_{i}+k\right) z_{i}^{j}-l z_{0}^{j}=0 \text { for } 1 \leq j \leq m+k-1+\sum_{i=1}^{n} p_{i}
$$

Since $m+k-1+\sum_{i=1}^{n} p_{i} \geq n+1$, it follows that the system of linear equations

$$
\sum_{i=0}^{n} z_{i}^{j} x_{i}=0, \quad 1 \leq j \leq n+1
$$

has a non-zero solution $\left(x_{0}, x_{1}, \ldots, x_{n}\right)=\left(-l, p_{1}+k, \ldots, p_{n}+k\right)$. This is impossible, as all $z_{i}$ are distinct.

Lemma 12 Let $k, m$ be positive integers, and let $f$ be a non-constant rational function. If $f(z)\left[f^{(k)}(z)-z^{m}\right] \neq 0$ for $z \neq 0$, and the multiplicity is at least $m+k+1$ when 0 is a zero of $f$, then $k=1$ and 0 is a zero of $f$ with exact multiplicity $2 m+2$.

Proof First, we show that $f$ cannot be a polynomial. Suppose not, then by $f(z) \neq 0$ for $z \neq 0, f(z)=C z^{s}$ for some constant $C \neq 0$ and integer $s \in \mathbb{N}$. Further, by the condition, $s \geq m+k+1$. Thus, $f^{(k)}(z)-z^{m}=A z^{s-k}-z^{m}=A z^{m}\left(z^{s-k-m}-1 / A\right)$, where $A \neq 0$ is a constant. This contradicts that $f^{(k)}(z)-z^{m} \neq 0$ for $z \neq 0$.

Thus, $f$ is a non-polynomial rational function. By Lemma 11, $f$ has at least one zero, and hence by $f(z) \neq 0$ for $z \neq 0$, we must have $f(0)=0$. Thus, we can write

$$
f(z)=C_{1} z^{l} \prod_{i=1}^{n}\left(z-z_{i}\right)^{-p_{i}}
$$

where $C_{1} \neq 0$ is constant, $z_{i} \in \mathbb{C}, 1 \leq i \leq n$ are distinct and non-zero, and $n, l, p_{i} \in$ $\mathbb{N}$ with $l \geq m+k+1$ (by the condition). Thus, 0 is a zero of $f^{(k)}$ with multiplicity $l-k \geq m+1$, and hence 0 is a zero of $f^{(k)}(z)-z^{m}$ with exact multiplicity $m$. Hence, since $f^{(k)}(z)-z^{m} \neq 0$ for $z \neq 0$, we have

$$
f^{(k)}(z)=z^{m}+C_{2} z^{m} \prod_{i=1}^{n}\left(z-z_{i}\right)^{-p_{i}-k},
$$

for some constant $C_{2} \neq 0$. However, by (12), one can obtain by induction that

$$
f^{(k)}(z)=C_{1} z^{l-k} P(z) \prod_{i=1}^{n}\left(z-z_{i}\right)^{-p_{i}-k}
$$

where

$$
P(z)=\prod_{j=0}^{k-1}\left(l-j-\sum_{i=1}^{n} p_{i}\right) z^{n k}+\cdots(\not \equiv 0)
$$


is a polynomial of degree at most $n k$. Thus by the two expressions of $f^{(k)}$ in (13) and (14),

$$
z^{m} \prod_{i=1}^{n}\left(z-z_{i}\right)^{p_{i}+k}+C_{2} z^{m}=C_{1} z^{l-k} P(z),
$$

and hence

$$
\prod_{i=1}^{n}\left(z-z_{i}\right)^{p_{i}+k}=-C_{2}+C_{1} z^{l-k-m} P(z) .
$$

Then, comparing the degrees of the both sides of (16) shows that

$$
\sum_{i=1}^{n}\left(p_{i}+k\right)=l-k-m+\operatorname{deg}(P) .
$$

Since $\operatorname{deg}(P) \leq n k$, it follows from (17) that $l \geq m+k+\sum_{i=1}^{n} p_{i}$. Thus by (15), $\operatorname{deg}(P)=n k$ and then by $(17)$,

$$
l=m+k+\sum_{i=1}^{n} p_{i}
$$

By (16), we also have

$$
C_{2}=-\prod_{i=1}^{n}\left(-z_{i}\right)^{p_{i}+k}
$$

Now by taking the logarithmic derivatives of (16), we have

$$
\sum_{i=1}^{n} \frac{p_{i}+k}{z-z_{i}}=O\left(z^{l-k-m-1}\right) \text { as } z \rightarrow 0
$$

Thus,

$$
\sum_{i=1}^{n} \frac{p_{i}+k}{\left(z_{i}\right)^{j}}=0 \text { for } 1 \leq j \leq l-k-m-1
$$

It follows that the system of linear equations

$$
\sum_{i=1}^{n} \frac{x_{i}}{\left(z_{i}\right)^{j}}=0, \quad 1 \leq j \leq l-k-m-1
$$


has a non-zero solution $\left(x_{1}, \ldots, x_{n}\right)=\left(p_{1}+k, \ldots, p_{n}+k\right)$. Since $z_{i}$ are non-zero and distinct, we get $l-k-m-1<n$, and hence by (18), $\sum_{i=1}^{n} p_{i}<n+1$. It follows that all $p_{i}=1$. Thus, $l=m+k+n$ and by (21),

$$
\sum_{i=1}^{n} \frac{1}{\left(z_{i}\right)^{j}}=0, \quad j=1, \ldots, n-1
$$

It follows from the well-known Newton's formula that $\prod_{i=1}^{n}\left(z-z_{i}\right)=z^{n}-r$, where $r \neq 0$ is a constant. Hence by (19)

$$
C_{2}=-\prod_{i=1}^{n}\left(-z_{i}\right)^{p_{i}+k}=-(-r)^{k+1}
$$

Thus by (12) and (13),

$$
\begin{aligned}
f(z) & =C_{1} z^{k+m+n}\left(z^{n}-r\right)^{-1}=C_{1} z^{k+m}\left(1-r z^{-n}\right)^{-1}, \\
f^{(k)}(z) & =z^{m}-(-r)^{k+1} z^{m}\left(z^{n}-r\right)^{-k-1} \\
& =z^{m}\left[1-\left(-r z^{-n}\right)^{k+1}\left(1-r z^{-n}\right)^{-k-1}\right] .
\end{aligned}
$$

By (25), we have

$$
f(z)=C_{1} z^{k+m} \sum_{s=0}^{\infty}\left(r z^{-n}\right)^{s}=C_{1} \sum_{s=0}^{\infty} r^{s} z^{k+m-s n}
$$

for $z$ satisfying $\left|r z^{-n}\right|<1$, and hence

$$
\begin{aligned}
f^{(k)}(z) & =C_{1} \sum_{s=0}^{\infty} r^{s} \prod_{j=1}^{k}(j+m-s n) z^{m-s n} \\
& =C_{1} z^{m} \sum_{s=0}^{\infty} \prod_{j=1}^{k}(j+m-s n)\left(r z^{-n}\right)^{s} .
\end{aligned}
$$

Thus by (26) and (27) with writing $w=r z^{-n}$,

$$
1-(-w)^{k+1}(1-w)^{-k-1}=C_{1} \sum_{s=0}^{\infty} \prod_{j=1}^{k}(j+m-s n) w^{s} \text { for }|w|<1
$$

Now comparing the coefficients of (28) yields that $\prod_{j=1}^{k}(j+m-s n)=0$ for $1 \leq s \leq k$. Thus, $s n-m \in\{1,2, \ldots, k\}$ for each $1 \leq s \leq k$. In particular, $1 \leq n-m \leq k$ and $1 \leq k n-m \leq k$. It follows that $k=1$ and $n=m+1$, and hence 0 is a zero of $f$ with exact multiplicity $l=m+k+n=2 m+2$. 


\section{Proof of Theorem 4}

Let $z_{0} \in D$ be a point and $\left\{f_{n}\right\} \subset \mathcal{F}$ be a sequence. We have to prove that $\left\{f_{n}\right\}$ has a subsequence which is normal at $z_{0}$. We may assume that $z_{0}=0$. Since $\left\{E_{f_{n}}\right\}$ are locally uniformly discrete in $D$, there exists $\delta_{0}>0$ such that $E_{f_{n}} \cap \Delta\left(0, \delta_{0}\right)$ contains at most one point $z_{f_{n}}$. That is to say, we have $f_{n} \neq 0$ and $f_{n}^{(k)} \neq h$ on $\Delta\left(0, \delta_{0}\right) \backslash\left\{z_{f_{n}}\right\}$ for all $f_{n}$. The following considerations for $\left\{f_{n}\right\}$ are understood to always hold with respect to the disk $\Delta\left(0, \delta_{0}\right)$.

Case 1 There exists $0<\delta<\delta_{0}$ such that $\left|z_{f_{n}}\right| \geq \delta$ for all $f_{n}$ (with $n$ sufficiently large). Then, we have $f_{n} \neq 0$ and $f_{n}^{(k)} \neq h$ on $\Delta(0, \delta)$ for all $f_{n}$. Thus by Theorem $1,\left\{f_{n}\right\}$ is normal on $\Delta(0, \delta)$, and hence at $z_{0}=0$.

Case 2 There exists a subsequence of $\left\{z_{f_{n}}\right\}$, which we continue to call $\left\{z_{f_{n}}\right\}$, such that $z_{f_{n}} \rightarrow z_{0}=0$. Then by Theorem $1,\left\{f_{n}\right\}$ is normal on $\Delta^{\circ}\left(0, \delta_{0}\right)$.

Suppose that $\left\{f_{n}\right\}$ has no subsequence which is normal at 0 . Next we consider two cases according to whether $h(0)$ is 0 or not.

Case 3 For the case $h(0) \neq 0$, we may say that $h(0)=1$. Since $\left\{f_{n}\right\}$ is not normal at 0 , by Lemma 5 , there exist a subsequence of $\left\{f_{n}\right\}$ which we continue to call $\left\{f_{n}\right\}$, a sequence of points $z_{n} \rightarrow 0$ and a sequence of positive numbers $\rho_{n} \rightarrow 0$ such that

$$
g_{n}(\zeta):=\rho_{n}^{-k} f_{n}\left(z_{n}+\rho_{n} \zeta\right) \rightarrow g(\zeta)
$$

spherically locally uniformly on $\mathbb{C}$, where $g$ is non-constant and meromorphic on $\mathbb{C}$.

Claim $1 g^{(k)}(\zeta) \not \equiv 1$. In fact, if $g^{(k)}(\zeta) \equiv 1$, then $g$ is a polynomial with exact degree $k$, so that $g$ has a zero $\zeta_{0} \in \mathbb{C}$ with multiplicity at most $k$, and hence by applying Hurwitz's theorem to (29), $g_{n}$ (for sufficiently large $n$ ) has a zero $\zeta_{n} \rightarrow \zeta_{0}$ with multiplicity at most $k$. It follows that $f_{n}$ has a zero $z_{n}+\rho_{n} \zeta_{n} \rightarrow 0$ with multiplicity at most $k$. This contradicts the assumption that all zeros of $f_{n}$ have multiplicity at least $k+2$.

Claim $2 g$ has at most one zero, and if it has, then the multiplicity is at least $k+2$.

The latter assertion follows from an argument similar to that in Claim 1. We now prove the former. Suppose that $g$ has at least two distinct zeros $\zeta_{1}, \zeta_{2} \in \mathbb{C}$. Then by applying Hurwitz's theorem to (29), $g_{n}$ (for sufficiently large $n$ ) has two distinct zeros $\zeta_{n}^{(1)}, \zeta_{n}^{(2)}$ tending to $\zeta_{1}, \zeta_{2}$ respectively, and hence $f_{n}$ has two distinct zeros $z_{n}+\rho_{n} \zeta_{n}^{(1)}$ and $z_{n}+\rho_{n} \zeta_{n}^{(2)}$, both tending to 0 . This contradicts that $f_{n}(z) \neq 0$ for $z \neq z_{f_{n}}$.

Claim $3 g^{(k)}-1$ has at most one zero.

By Claim 1 and the fact that

$$
f_{n}^{(k)}\left(z_{n}+\rho_{n} \zeta\right)-h\left(z_{n}+\rho_{n} \zeta\right)=g_{n}^{(k)}(\zeta)-h\left(z_{n}+\rho_{n} \zeta\right) \rightarrow g^{(k)}(\zeta)-1(\not \equiv 0)
$$

locally uniformly on $\mathbb{C} \backslash g^{-1}(\infty)$, an argument similar to that in Claim 2 yields this claim. 
Claim 4 Either $g \neq 0$ or $g^{(k)} \neq 1$.

Suppose not, say $g\left(\zeta_{0}^{(1)}\right)=0$ and $g^{(k)}\left(\zeta_{0}^{(2)}\right)=1$. Since $\zeta_{0}^{(1)}$ is a zero of $g$ with multiplicity $\geq k+2$, we have $g^{(k)}\left(\zeta_{0}^{(1)}\right)=0$, and hence $\zeta_{0}^{(1)} \neq \zeta_{0}^{(2)}$. By applying Hurwitz's theorem to (29) and (30), there exist points $\zeta_{n}^{(i)} \rightarrow \zeta_{0}^{(i)}$ such that $g_{n}\left(\zeta_{n}^{(1)}\right)=$ 0 and $g_{n}^{(k)}\left(\zeta_{n}^{(2)}\right)-h\left(z_{n}+\rho_{n} \zeta_{n}^{(2)}\right)=0$, and hence $f_{n}\left(z_{n}+\rho_{n} \zeta_{n}^{(1)}\right)=0$ and $f_{n}^{(k)}\left(z_{n}+\right.$ $\left.\rho_{n} \zeta_{n}^{(2)}\right)-h\left(z_{n}+\rho_{n} \zeta_{n}^{(2)}\right)=0$. Since $f_{n}(z) \neq 0$ and $f_{n}^{(k)}(z) \neq h(z)$ for $z \neq z_{f_{n}}$, it follows that $z_{n}+\rho_{n} \zeta_{n}^{(1)}=z_{n}+\rho_{n} \zeta_{n}^{(2)}\left(=z_{f_{n}}\right)$ so that $\zeta_{n}^{(1)}=\zeta_{n}^{(2)}$, and hence $\zeta_{0}^{(1)}=\zeta_{0}^{(2)}$. This is a contradiction.

Thus by Claims 2-4, $g\left(g^{(k)}-1\right)$ has at most one zero, and hence by Lemma $6, g$ is a rational function. Further by Lemma 9, g has a zero with multiplicity at most $k+1$. This contradicts Claim 2 which says that the zero of $g$ has multiplicity at least $k+2$.

Case 4 Next we consider the case that $h(0)=0$. Then $h(z)=z^{m} \phi(z)$, where $m \in$ $\mathbb{N}$ and $\phi$ is holomorphic with $\phi(0) \neq 0$. We can say $\phi \neq 0$ in $\Delta\left(0, \delta_{0}\right)$ with the normalization $\phi(0)=1$. Set for each $n$

$$
F_{n}(z)=z^{-m} f_{n}(z)
$$

Then $F_{n}(z) \neq 0$ for $z \neq z_{f_{n}}$, since $f_{n}(z) \neq 0$ for $z \neq z_{f_{n}}$.

We first show that $\left\{F_{n}\right\}$ has no subsequence which is normal at 0 . Suppose not, say $\left\{F_{n}\right\}$ is normal at 0 . Then $\left\{F_{n}\right\}$ has a subsequence, which we continue to call $\left\{F_{n}\right\}$, such that $\left\{F_{n}\right\}$ converges spherically locally uniformly to $\psi$ which may be $\infty$ identically in some neighborhood $\Delta\left(0, \eta_{0}\right)$.

If $\psi(0) \neq \infty$, then for sufficiently large $n, f_{n}(z) \neq \infty$ and $\left|F_{n}(z)\right| \leq M$ in some closed domain $\bar{\Delta}(0, \eta)$ with $\eta<\eta_{0}$, where $M>0$ is a constant. It follows that the functions $f_{n}$ are holomorphic and satisfy $\left|f_{n}(z)\right| \leq M|z|^{m}$ on $\bar{\Delta}(0, \eta)$. By the Montel's theorem, $\left\{f_{n}\right\}$ is normal at 0 , which contradicts our assumption that $\left\{f_{n}\right\}$ is not normal at 0 .

If $\psi(0)=\infty$, then for sufficiently large $n,\left|F_{n}(z)\right|>1$ in some closed domain $\bar{\Delta}(0, \eta)$ with $\eta<\eta_{0}$. We claim that $f_{n}\left(z_{f_{n}}\right) \neq 0$ for all (sufficiently large) $n$. In fact, if $f_{n}\left(z_{f_{n}}\right)=0$, then as $F_{n}\left(z_{f_{n}}\right) \neq 0$, we see that $z_{f_{n}}=0$, and so by the assumption on the multiplicities of common zeros of $f_{n}$ and $h, z_{f_{n}}=0$ is a zero of $f_{n}$ with multiplicity at least $m+k+1>m$, and hence $F_{n}(0)=0$, which contradicts that $\left|F_{n}(z)\right|>1$. Thus, $f_{n}\left(z_{f_{n}}\right) \neq 0$. This, combined with the fact that $f_{n}(z) \neq 0$ for $z \neq z_{f_{n}}$, shows that the functions $1 / f_{n}$ are holomorphic. By $\left|F_{n}(z)\right|>1$, we have $\left|1 / f_{n}(z)\right|<|z|^{-m}$ on $\bar{\Delta}(0, \eta)$. Now the maximum modulus principle implies that $\left|1 / f_{n}(z)\right| \leq \eta^{-m}$ on $\bar{\Delta}(0, \eta)$ and hence $\left\{1 / f_{n}\right\}$ is normal at 0 by Montel's theorem. This again contradicts our assumption that $\left\{f_{n}\right\}$ is not normal at 0 .

Thus, $\left\{F_{n}\right\}$ has no subsequence which is normal at 0 .

Claim 5 If $z_{f_{n}}$ is a zero of $F_{n}$, then the multiplicity is at least $k+1$, or $k+2$ if $z_{f_{n}} \neq 0$. In fact, suppose $F_{n}\left(z_{f_{n}}\right)=0$, then $f_{n}\left(z_{f_{n}}\right)=0$. Thus for the case $z_{f_{n}} \neq 0$, the claim is true by the assumption on the multiplicities of the zeros of $f_{n}$, while for the case $z_{f_{n}}=0, z_{f_{n}}=0$ is a common zero of $f_{n}$ and $h$, and hence by the assumption on the multiplicities of the common zeros of $f_{n}$ and $h, 0$ is a zero of $f_{n}$ with multiplicity at least $k+m+1$, and then the claim follows. 
Thus by Claim 5 and the fact that $F_{n}(z) \neq 0$ for $z \neq z_{f_{n}}$, Lemma 5 can be applied, and so there exist a subsequence of $\left\{f_{n}\right\}$, which we continue to call $\left\{f_{n}\right\}$, a sequence of points $w_{n} \rightarrow 0$ and a sequence of positive numbers $\eta_{n} \rightarrow 0$ such that

$$
\widehat{g}_{n}(\zeta):=\eta_{n}^{-k} F_{n}\left(w_{n}+\eta_{n} \zeta\right)=\eta_{n}^{-k}\left(w_{n}+\eta_{n} \zeta\right)^{-m} f_{n}\left(w_{n}+\eta_{n} \zeta\right) \rightarrow \widehat{g}(\zeta)
$$

spherically locally uniformly on $\mathbb{C}$, where $\widehat{g}$ is non-constant and meromorphic on $\mathbb{C}$.

Claim $6 \widehat{g}$ has at most one zero.

In fact, if $\widehat{g}$ has two distinct zeros $\zeta_{1}, \zeta_{2} \in \mathbb{C}$, then applying Hurwitz's theorem to (32), there exist points $\zeta_{i, n} \rightarrow \zeta_{i}$ such that $\widehat{g}_{n}\left(\zeta_{i, n}\right)=0$ and hence $F_{n}\left(w_{n}+\eta_{n} \zeta_{i, n}\right)=0$. Since $F_{n}(z) \neq 0$ for $z \neq z_{f_{n}}$, we get $w_{n}+\eta_{n} \zeta_{1, n}=w_{n}+\eta_{n} \zeta_{2, n}\left(=z_{f_{n}}\right)$. It follows that $\zeta_{1, n}=\zeta_{2, n}$, and hence $\zeta_{1}=\zeta_{2}$. This is a contradiction.

Next we consider two subcases according to whether the sequence $\left\{w_{n} / \eta_{n}\right\}$ is bounded or unbounded.

Case 5 First assume that the sequence $\left\{w_{n} / \eta_{n}\right\}$ is unbounded. Then, there exists a subsequence, which we continue to call $\left\{w_{n} / \eta_{n}\right\}$, such that $w_{n} / \eta_{n} \rightarrow \infty$.

Claim 7 If $\widehat{g}$ has a zero, then the multiplicity is at least $k+2$. And hence, $\widehat{g}^{(k)} \not \equiv 1$.

In fact, if $\widehat{g}\left(\zeta_{0}\right)=0$, then as above, there exist points $\zeta_{n} \rightarrow \zeta_{0}$ such that $F_{n}\left(w_{n}+\right.$ $\left.\eta_{n} \zeta_{n}\right)=0$, and hence $z_{f_{n}}=w_{n}+\eta_{n} \zeta_{n}$. As $w_{n} / \eta_{n} \rightarrow \infty$, we see that $z_{f_{n}} \neq 0$, and hence by Claim $5, z_{f_{n}}$ is a zero of $F_{n}$ with multiplicity at least $k+2$. Thus, $\zeta_{n}$ is a zero of $\widehat{g}_{n}$ with multiplicity at least $k+2$. The claim then follows.

Claim $8 \widehat{g}^{(k)}-1$ has at most one zero.

This follows from a similar argument as above with the following fact that

$$
\begin{aligned}
\frac{f_{n}^{(k)}\left(w_{n}+\eta_{n} \zeta\right)}{h\left(w_{n}+\eta_{n} \zeta\right)} & =\frac{\left[\left(\eta_{n}\right)^{-k} f_{n}\left(w_{n}+\eta_{n} \zeta\right)\right]^{(k)}}{h\left(w_{n}+\eta_{n} \zeta\right)}=\frac{\left[\left(w_{n}+\eta_{n} \zeta\right)^{m} \widehat{g}_{n}(\zeta)\right]^{(k)}}{h\left(w_{n}+\eta_{n} \zeta\right)} \\
& =\frac{\left[\left(\zeta+\frac{w_{n}}{\eta_{n}}\right)^{m} \widehat{g}_{n}(\zeta)\right]^{(k)}}{\left(\zeta+\frac{w_{n}}{\eta_{n}}\right)^{m} \phi\left(w_{n}+\eta_{n} \zeta\right)}=\frac{\sum_{i=0}^{k}\left(\begin{array}{c}
k \\
i
\end{array}\right)\left[\left(\zeta+\frac{w_{n}}{\eta_{n}}\right)^{m}\right]^{(k-i)} \widehat{g}_{n}^{(i)}(\zeta)}{\left(\zeta+\frac{w_{n}}{\eta_{n}}\right)^{m} \phi\left(w_{n}+\eta_{n} \zeta\right)} \\
& =\frac{1}{\phi\left(w_{n}+\eta_{n} \zeta\right)} \sum_{i=0}^{k} \frac{C_{i} \widehat{g}_{n}^{(i)}(\zeta)}{\left(\zeta+\frac{w_{n}}{\eta_{n}}\right)^{k-i}} \rightarrow \widehat{g}^{(k)}(\zeta)
\end{aligned}
$$

locally uniformly on $\mathbb{C} \backslash \widehat{g}^{-1}(\infty)$, where $C_{i}=m(m-1) \cdots(m-(k-i)+1)\left(\begin{array}{l}k \\ i\end{array}\right)$ are constants, and in particular, $C_{k}=1$.

Claim 9 Either $\widehat{g} \neq 0$ or $\widehat{g}^{(k)} \neq 1$.

Combined with the fact that $F_{n}(z) \neq 0$ and $f_{n}^{(k)}(z) \neq h(z)$ for $z \neq z_{f_{n}}$, this can be shown by an argument similar to the proof of Claim 4 by applying Hurwitz's theorem to (32) and (33).

However, as in Case 2.1, the above Claims 6-9 lead to a contradiction. 
Case 6 Now we consider the case that $\left\{w_{n} / \eta_{n}\right\}$ is bounded. Then, there is a subsequence, which we continue to call $\left\{w_{n} / \eta_{n}\right\}$, such that $w_{n} / \eta_{n} \rightarrow \alpha \in \mathbb{C}$. It follows from (32) that

$$
\frac{f_{n}\left(\eta_{n} \zeta\right)}{\eta_{n}^{k+m} \zeta^{m}}=\widehat{g}_{n}\left(\zeta-\frac{w_{n}}{\eta_{n}}\right) \rightarrow \widehat{g}(\zeta-\alpha)
$$

spherically locally uniformly on $\mathbb{C}$, and hence

$$
G_{n}(\zeta):=\frac{f_{n}\left(\eta_{n} \zeta\right)}{\eta_{n}^{k+m}}=\zeta^{m} \cdot \frac{f_{n}\left(\eta_{n} \zeta\right)}{\eta_{n}^{k+m} \zeta^{m}} \rightarrow G(\zeta):=\zeta^{m} \widehat{g}(\zeta-\alpha)
$$

spherically locally uniformly on $\mathbb{C}^{*}=\mathbb{C} \backslash\{0\}$, or on $\mathbb{C}$ if $\widehat{g}(-\alpha) \neq \infty$. Obviously, $G$ is meromorphic on $\mathbb{C}$ and $G \not \equiv 0$.

Claim 10 G is non-constant. Suppose not. Then, $\widehat{g}(\zeta-\alpha)=A \zeta^{-m}$ for some non-zero constant A. Thus by (34), we see that

$$
G_{n}(\zeta):=\frac{f_{n}\left(\eta_{n} \zeta\right)}{\eta_{n}^{k+m}} \rightarrow A
$$

locally uniformly on $\mathbb{C}$. Hence $G_{n}^{(k)}(\zeta) \rightarrow 0$, so that

$$
\eta_{n}^{-m}\left[f_{n}^{(k)}\left(\eta_{n} \zeta\right)-h\left(\eta_{n} \zeta\right)\right]=G_{n}^{(k)}(\zeta)-\zeta^{m} \phi\left(\eta_{n} \zeta\right) \rightarrow-\zeta^{m}
$$

locally uniformly on $\mathbb{C}$. Thus by applying Hurwitz's theorem to (37), there exist exactly $m$ points $\zeta_{n}^{(j)} \rightarrow 0, j=1,2, \ldots, m$, such that $f_{n}^{(k)}\left(\eta_{n} \zeta_{n}^{(j)}\right)=h\left(\eta_{n} \zeta_{n}^{(j)}\right)$. It follows from $f_{n}^{(k)}(z) \neq h(z)$ for $z \neq z_{f_{n}}$ that $z_{f_{n}}=\eta_{n} \zeta_{n}^{(j)}$ for all $j$. This shows that the $m$ points $\zeta_{n}^{(j)}$ are coincide with $\zeta_{n}:=z_{f_{n}} / \eta_{n}$, and $z_{f_{n}}$ is a zero of $f_{n}^{(k)}(z)-h(z)$ with multiplicity $m$, and by (36), $f_{n}\left(z_{f_{n}}\right) \neq 0$. Thus, $f_{n}(z) \neq 0$ for $z \in \Delta\left(0, \delta_{0}\right)$.

Since $\left\{f_{n}\right\}$ is normal on $\Delta^{\circ}\left(0, \delta_{0}\right)$, but not normal at 0 , it follows from $f_{n} \neq 0$ on $\Delta\left(0, \delta_{0}\right)$ that there exists a subsequence of $\left\{f_{n}\right\}$, which we continue to call $\left\{f_{n}\right\}$, such that $f_{n} \rightarrow 0$ and hence $f_{n}^{(k)} \rightarrow 0$ locally uniformly on $\Delta^{\circ}\left(0, \delta_{0}\right)$.

Thus by the argument principle, we see that

$$
n\left(\frac{\delta_{0}}{2}, f_{n}^{(k)}-h\right)-n\left(\frac{\delta_{0}}{2}, \frac{1}{f_{n}^{(k)}-h}\right) \rightarrow n\left(\frac{\delta_{0}}{2}, h\right)-n\left(\frac{\delta_{0}}{2}, \frac{1}{h}\right)=-m,
$$

where $n(r, f)$ is the number of poles of $f$ in $\Delta(0, r)$, and $n(r, 1 / f)$ is the number of zeros of $f$ in $\Delta(0, r)$. Since both hand sides of (38) are integers, we see that for sufficiently large $n$,

$$
n\left(\frac{\delta_{0}}{2}, f_{n}^{(k)}-h\right)=n\left(\frac{\delta_{0}}{2}, \frac{1}{f_{n}^{(k)}-h}\right)-m=0
$$


It follows from (39) that $f_{n}$ are holomorphic on $\Delta\left(0, \delta_{0} / 2\right)$. Hence by $f_{n} \rightarrow 0$ locally uniformly on $\Delta^{\circ}\left(0, \delta_{0}\right)$, we get $f_{n} \rightarrow 0$ locally uniformly on $\Delta\left(0, \delta_{0}\right)$. This contradicts that $\left\{f_{n}\right\}$ is not normal at 0 .

Claim $11 G$ has at most one zero on $\mathbb{C}^{*}$, or on $\mathbb{C}$ if $\widehat{g}(-\alpha) \neq \infty$.

This can be proved by applying Hurwitz's theorem to (35) with the fact that $f_{n}(z) \neq$ 0 for $z \neq z_{f_{n}}$.

Claim 12 If $G$ has a zero on $\mathbb{C}^{*}$, then $z_{f_{n}} \neq 0$ and the multiplicity of the zero of $G$ on $\mathbb{C}^{*}$ is at least $k+2$, or $k+3$ if $m=1$.

Suppose that $\zeta_{0} \in \mathbb{C}^{*}$ is a zero of $G$. Then by applying Hurwitz's theorem to (35), $G_{n}$ has a zero $\zeta_{n} \rightarrow \zeta_{0}$. Since $\zeta_{0} \neq 0, \zeta_{n} \neq 0$ (for $n$ large enough). It follows that $\eta_{n} \zeta_{n} \rightarrow 0$ is a non-zero zero of $f_{n}$. Since $f_{n}(z) \neq 0$ for $z \neq z_{f_{n}}$, we see that $z_{f_{n}}=\eta_{n} \zeta_{n} \neq 0$. Hence by the assumption, $z_{f_{n}}$ is a zero of $f_{n}$ with multiplicity at least $k+2$, or $k+3$ if $m=1$. It follows that the multiplicity of the zero $\zeta_{0}$ of $G$ is at least $k+2$, or $k+3$ if $m=1$.

Claim 13 If $G(0)=0$, then $f_{n}(0)=0$ and hence $z_{f_{n}}=0$ (for sufficiently large $\left.n\right)$.

For otherwise, say $f_{n}(0) \neq 0$. Then by (34), 0 is a pole of $\widehat{g}_{n}\left(\zeta-w_{n} / \eta_{n}\right)$ with multiplicity at least $m$, and hence is a pole of $\widehat{g}(\zeta-\alpha)$ with multiplicity at least $m$. This shows that 0 is not a zero of $G(\zeta)=\zeta^{m} \widehat{g}(\zeta-\alpha)$, which is a contradiction.

By Claims 12 and 13, we see that if $G(0)=0$, then $G(\zeta) \neq 0$ for $\zeta \in \mathbb{C}^{*}$. Hence by Claim 11, G has at most one zero on $\mathbb{C}$.

Claim 14 If 0 is a zero of $G$, then the multiplicity is at least $m+k+1$ for $k>1$ and $2 m+3$ for $k=1$.

In fact, if 0 is a zero of $G$, then Claim 13 shows that 0 is a common zero of $f_{n}$ and $h$. Let $m_{f_{n}}$ be the multiplicity of 0 as a zero of $f_{n}$. Then by the assumption, $m_{f_{n}} \geq m+k+1$ for $k>1$ and $m_{f_{n}} \geq 2 m+3$ for $k=1$. It follows from (34) that $-w_{n} / \eta_{n}$ is a zero of $\widehat{g}_{n}$ with multiplicity $m_{f_{n}}-m$, so that $-\alpha$ is a zero of $\widehat{g}$ with multiplicity at least $k+1$ for $k>1$ and $m+3$ for $k=1$. The assertion then follows.

Now by (35), we have

$$
\eta_{n}^{-m}\left[f_{n}^{(k)}\left(\eta_{n} \zeta\right)-h\left(\eta_{n} \zeta\right)\right]=G_{n}^{(k)}(\zeta)-\zeta^{m} \phi\left(\eta_{n} \zeta\right) \rightarrow G^{(k)}(\zeta)-\zeta^{m}
$$

locally uniformly on $\mathbb{C}^{*} \backslash G^{-1}(\infty)$, or on $\mathbb{C} \backslash G^{-1}(\infty)$ if $\widehat{g}(-\alpha) \neq \infty$.

Claim $15 G^{(k)}(\zeta) \not \equiv \zeta^{m}$.

For otherwise, $G(\zeta)=c \zeta^{m+k}+P(\zeta)$ for some constant $c \neq 0$ and polynomial $P$ of degree at most $k-1$. Thus, $G$ has at least one zero in $\mathbb{C}$. Let $\zeta_{0}$ be a zero of $G$, then by the fact that $\zeta_{0}$ has multiplicity $>k$, we get $G^{(k)}\left(\zeta_{0}\right)=0$ and hence $\zeta_{0}=0$ by $G^{(k)}(\zeta) \equiv \zeta^{m}$. It follows that $G(\zeta)=c^{\prime} \zeta^{l}$ for some $c^{\prime} \in \mathbb{C}$ and $l \in \mathbb{N}$. Thus, $c \zeta^{m+k}+P(\zeta)=c^{\prime} \zeta^{l}$. By comparing the degrees and coefficients, we see that $P \equiv 0$. Thus, $G(\zeta)=c \zeta^{m+k}$. This contradicts Claim 14 which says that the multiplicity is at least $m+k+1$ if 0 is a zero of $G$.

Claim $16 G^{(k)}(\zeta)-\zeta^{m}$ has at most one zero on $\mathbb{C}^{*}$, or on $\mathbb{C}$ if $\widehat{g}(-\alpha) \neq \infty$.

By Claim 15, this claim can be seen by applying Hurwitz's theorem to (40) with the condition $f_{n}^{(k)}(z) \neq h(z)$ for $z \neq z_{f_{n}}$. 
By Claims 10, 11 and 16, it follows from Lemma 6 that $G$ is a non-constant rational function.

Claim 17 If $G^{(k)}(0)=0$, then $G \neq 0$ on $\mathbb{C}^{*}$.

Suppose that $G\left(\zeta_{0}\right)=0$ for some $\zeta_{0} \neq 0$. Then $G$ is holomorphic at 0 . Since we have proved before Claim 14 that $G$ has at most one zero on $\mathbb{C}$, we get $G(0) \neq 0$. Thus by $G(0) \neq 0, \infty$, it follows from $G(\zeta)=\zeta^{m} \widehat{g}(\zeta-\alpha)$ that $\widehat{g}(-\alpha)=\infty$ and that 0 is a pole of $\widehat{g}(\zeta-\alpha)$ with exact multiplicity $m$. Thus by (34), there exists a positive number $\delta$ such that $f_{n}\left(\eta_{n} \zeta\right) \neq \infty$ for $|\zeta| \leq \delta$ (and n large enough), and hence $G_{n}(\zeta)$ is holomorphic on $|\zeta| \leq \delta$. Thus, on $|\zeta| \leq \delta$, the convergence of (40) is uniformly, and then Hurwitz's theorem can be applied to (40). Since $G^{(k)}(0)=0^{m}$ and $G^{(k)}(\zeta) \not \equiv \zeta^{m}$ by Claim 15, there exist points $\zeta_{n} \rightarrow 0$ such that $G_{n}^{(k)}\left(\zeta_{n}\right)=\zeta_{n}^{m} \phi\left(\eta_{n} \zeta_{n}\right)$ so that $f_{n}^{(k)}\left(\eta_{n} \zeta_{n}\right)=h\left(\eta_{n} \zeta_{n}\right)$. Hence, by $f_{n}^{(k)}(z) \neq h(z)$ for $z \neq z_{f_{n}}$, we get $z_{f_{n}}=\eta_{n} \zeta_{n}$. On the other hand, since $G\left(\zeta_{0}\right)=0$, applying Hurwitz's theorem to (35) yields that there exist points $\zeta_{n}^{\prime} \rightarrow \zeta_{0}$ such that $G_{n}\left(\zeta_{n}^{\prime}\right)=0$ so that $f_{n}\left(\eta_{n} \zeta_{n}^{\prime}\right)=0$. Thus by $f_{n}(z) \neq 0$ for $z \neq z_{f_{n}}$, we get $z_{f_{n}}=\eta_{n} \zeta_{n}^{\prime}$. Hence $\eta_{n} \zeta_{n}=\eta_{n} \zeta_{n}^{\prime}\left(=z_{f_{n}}\right)$ and then $\zeta_{n}^{\prime}=\zeta_{n}$. It follows that $\zeta_{0}=0$, which is a contradiction.

Claim 18 If $G^{(k)}(0)=0$, then $G^{(k)}(\zeta)-\zeta^{m} \neq 0$ on $\mathbb{C}^{*}$.

Suppose that $G^{(k)}\left(\zeta_{0}\right)=\zeta_{0}^{m}$ for some $\zeta_{0} \neq 0$. Then $G$ is holomorphic at 0 . If $G(0)=0$, then by Claim $14, \widehat{g}(-\alpha)=0$, and hence by Claim $16, G^{(k)}(\zeta)-\zeta^{m}$ has at most one zero on $\mathbb{C}$. This contradicts that $G^{(k)}(0)=0^{m}$ and $G^{(k)}\left(\zeta_{0}\right)=\zeta_{0}^{m}$. Thus, $G(0) \neq 0$. Now by an argument similar to that in the proof of Claim 17, there exists $\delta>0$ such that on $|\zeta| \leq \delta$, the convergence of (40) is uniformly, and then Hurwitz's theorem can be applied to (40). It follows from $G^{(k)}(0)=0^{m}$ that there exist points $\zeta_{n} \rightarrow 0$ such that $G_{n}^{(k)}\left(\zeta_{n}\right)=\zeta_{n}^{m} \phi\left(\eta_{n} \zeta_{n}\right)$ so that $f_{n}^{(k)}\left(\eta_{n} \zeta_{n}\right)=h\left(\eta_{n} \zeta_{n}\right)$. Thus by $f_{n}^{(k)}(z)=h(z)$ for $z \neq z_{f_{n}}$, we get $z_{f_{n}}=\eta_{n} \zeta_{n}$. On the other hand, since $G^{(k)}\left(\zeta_{0}\right)=\zeta_{0}^{m}$ with $\zeta_{0} \neq 0$, by applying Hurwitz's theorem to (40), there exist points $\zeta_{n}^{\prime} \rightarrow \zeta_{0}$ such that $G_{n}^{(k)}\left(\zeta_{n}^{\prime}\right)=\left(\zeta_{n}^{\prime}\right)^{m} \phi\left(\eta_{n} \zeta_{n}^{\prime}\right)$ so that $f_{n}^{(k)}\left(\eta_{n} \zeta_{n}^{\prime}\right)=h\left(\eta_{n} \zeta_{n}^{\prime}\right)$. Again by $f_{n}^{(k)}(z)=h(z)$ for $z \neq z_{f_{n}}$, we get $z_{f_{n}}=\eta_{n} \zeta_{n}^{\prime}$. Thus $\eta_{n} \zeta_{n}=\eta_{n} \zeta_{n}^{\prime}\left(=z_{f_{n}}\right)$ and then $\zeta_{n}^{\prime}=\zeta_{n}$. It follows that $\zeta_{0}=0$, which is a contradiction.

Thus by Claims 16 and $18, G^{(k)}(\zeta)-\zeta^{m}$ has at most one zero on $\mathbb{C}$.

Claim 19 At least one of $G$ and $G^{(k)}(\zeta)-\zeta^{m}$ has no zero on $\mathbb{C}^{*}$.

Suppose that $G\left(\zeta_{0}^{(1)}\right)=0$ and $G^{(k)}\left(\zeta_{0}^{(2)}\right)=\left(\zeta_{0}^{(2)}\right)^{m}$ for some $\zeta_{0}^{(1)}, \zeta_{0}^{(2)} \in \mathbb{C}^{*}$. By Claim $12, \zeta_{0}^{(1)}$ is a zero of $G$ with multiplicity $\geq k+2$, and hence $G^{(k)}\left(\zeta_{0}^{(1)}\right)=0$. It follows that $\zeta_{0}^{(1)} \neq \zeta_{0}^{(2)}$. Applying Hurwitz's theorem to (35) shows that there exist points $\zeta_{n}^{(1)} \rightarrow \zeta_{0}^{(1)}$ such that $G_{n}\left(\zeta_{n}^{(1)}\right)=0$. Thus $f_{n}\left(\eta_{n} \zeta_{n}^{(1)}\right)=0$, and hence by $f_{n}(z) \neq 0$ for $z \neq z_{f_{n}}$, we get $z_{f_{n}}=\eta_{n} \zeta_{n}^{(1)}$. Applying Hurwitz's theorem to (40) shows that there exist points $\zeta_{n}^{(2)} \rightarrow \zeta_{0}^{(2)}$ such that $f_{n}^{(k)}\left(\eta_{n} \zeta_{n}^{(2)}\right)=h\left(\eta_{n} \zeta_{n}^{(2)}\right)$, and hence by $f_{n}^{(k)}(z) \neq h(z)$ for $z \neq z_{f_{n}}$, we get $z_{f_{n}}=\eta_{n} \zeta_{n}^{(2)}$. Thus $\zeta_{n}^{(1)}=\zeta_{n}^{(2)}$ and hence $\zeta_{0}^{(1)}=\zeta_{0}^{(2)}$. This contradicts that $\zeta_{0}^{(1)} \neq \zeta_{0}^{(2)}$.

Now, according to Claim 19, we are in one of the following three cases. Note that we have proved before Claim 17 that $G$ is a non-constant rational function. 
Case 7 Neither of $G$ and $G^{(k)}(\zeta)-\zeta^{m}$ has a zero on $\mathbb{C}^{*}$.

Then by Lemma $11, G$ has at least one zero on $\mathbb{C}$, and hence $G(0)=0$. It now follows from Claim 14 and Lemma 12 that Case A cannot occur.

Case $8 G\left(\zeta_{0}\right)=0$ for some $\zeta_{0} \in \mathbb{C}^{*}$ and $G^{(k)}(\zeta)-\zeta^{m}$ has no zero on $\mathbb{C}^{*}$.

Then $G(\zeta) \neq 0$ for $\zeta \in \mathbb{C} \backslash\left\{\zeta_{0}\right\}$, since we have proved before Claim 14 that $G$ has at most one zero on $\mathbb{C}$. Also, by Claim 17 , we have $G^{(k)}(0) \neq 0$, and hence $G^{(k)}(\zeta) \neq \zeta^{m}$ for $\zeta \in \mathbb{C}$. It now follows from Claim 12 and Lemma 10 that Case B cannot occur.

Case $9 G^{(k)}\left(\zeta_{0}\right)=\zeta_{0}^{m}$ for some $\zeta_{0} \in \mathbb{C}^{*}$ and $G(\zeta)$ has no zero on $\mathbb{C}^{*}$.

Then $G^{(k)}(\zeta) \neq \zeta^{m}$ for $\zeta \in \mathbb{C} \backslash\left\{\zeta_{0}\right\}$, since we have proved before Claim 19 that $G^{(k)}(\zeta)-\zeta^{m}$ has at most one zero on $\mathbb{C}$. Hence by Lemma $11, G$ has at least one zero on $\mathbb{C}$, so that we must have $G(0)=0$. It now follows from Claim 14 that $G^{(k)}(0)=0$, which contradicts that $G^{(k)}(\zeta) \neq \zeta^{m}$ for $\zeta \in \mathbb{C} \backslash\left\{\zeta_{0}\right\}$.

The proof of Theorem 4 is completed.

\section{Proof of Theorem 3}

From the proof of Theorem 4, we have the following result, which shows that the multiplicity $k+3$ in Theorem 4 can be replaced by $k+2$ when the set $E_{f}$ is independent of $f$. Note that the assumption that multiplicity $\geq k+3$ in Theorem 4 is only used to rule out the Case B within the proof of Theorem 4 .

Theorem 13 Let $k \in \mathbb{N}$ and $h(\not \equiv 0)$ be a function holomorphic on $D$. Let $\mathcal{F}$ be a family of meromorphic functions in $D$, all of whose zeros have multiplicity at least $k+2$. Suppose that there exists a point $z_{0} \in D$ such that on $D \backslash\left\{z_{0}\right\}, f \neq 0$ and $f^{(k)} \neq h$ for all functions $f \in \mathcal{F}$. Suppose additionally that if $z_{0}$ is a common zero of $f \in \mathcal{F}$ and $h$, the multiplicities $m_{f}$ for $f$ and $m_{h}$ for $h$ satisfy $m_{f} \geq m_{h}+k+1$ for $k>1$ and $m_{f} \geq 2 m_{h}+3$ for $k=1$. Then $\mathcal{F}$ is normal in $D$.

Proof The proof is similar to, and more simple than, the proof of Theorem 4, since in this case, we only have to consider the case that $z f_{n}=0$ for all $n$ in the proof of Theorem 4. In fact, since $z_{f_{n}}=0$, we see from Claim 12 that $G$ has no zeros on $\mathbb{C}^{*}$, and a similar argument by applying Hurwitz's theorem to (40) can be used to show that $G^{(k)}(\zeta)-\zeta^{m}$ has no zeros on $\mathbb{C}^{*}$. So only the Case A appears and is required to be ruled out.

Proof of Theorem 3 Let $z_{0} \in D$ and a sequence $\left\{f_{n}\right\} \subset \mathcal{F}$. We have to prove that $\left\{f_{n}\right\}$ is normal at $z_{0}$.

Suppose first that there exists a neighborhood $U_{0}$ of $z_{0}$ such that on $U_{0} \backslash\left\{z_{0}\right\}$, $f_{1} \neq 0$ and $f_{1}^{(k)} \neq h$. Then by the condition, on $U_{0} \backslash\left\{z_{0}\right\}, f_{n} \neq 0$ and $f_{n}^{(k)} \neq h$ for all functions $f_{n}$. Hence, by Theorem $13,\left\{f_{n}\right\}$ is normal in $U_{0}$ and hence normal at $z_{0}$.

The other case is that there exists a sequence $z_{n} \rightarrow z_{0}, z_{n} \neq z_{0}$ such that either $f_{1}\left(z_{n}\right)=0$ or $f_{1}^{(k)}\left(z_{n}\right)=h\left(z_{n}\right)$. It follows that either $f_{1}(z) \equiv 0$ or $f_{1}^{(k)}(z) \equiv h(z)$. Thus by the condition, either $f_{n}(z) \equiv 0$ or $f_{n}^{(k)}(z) \equiv h(z)$ for all $n$. 
It is obviously that $\left\{f_{n}\right\}$ is normal if $f_{n}(z) \equiv 0$. Thus, we consider the case that $f_{n}^{(k)}(z) \equiv h(z)$. If $\left\{f_{n}\right\}$ is not normal at $z_{0}$, then by Lemma 5 , there exists points $z_{n} \rightarrow z_{0}$, positive numbers $\rho_{n} \rightarrow 0$ and functions $f_{n} \in\left\{f_{n}\right\}$ such that $g_{n}(\zeta)=f_{n}\left(z_{n}+\rho_{n} \zeta\right) \rightarrow g(\zeta)$ locally uniformly on $\mathbb{C}$, where $g$ is a non-constant meromorphic function, all of whose zeros have multiplicity at least $k+2$. Since $g_{n}^{(k)}(\zeta)=\rho_{n}^{k} f_{n}^{(k)}\left(z_{n}+\rho_{n} \zeta\right)=\rho_{n}^{k} h\left(z_{n}+\rho_{n} \zeta\right) \rightarrow 0$, we get $g^{(k)}(\zeta) \equiv 0$. Since all zeros of $g$ have multiplicity at least $k+2$, it follows that $g$ is a constant, which is a contradiction.

Thus, $\left\{f_{n}\right\}$ is normal at $z_{0}$. Theorem 3 is proved.

Acknowledgments The author would like to thank the referee for his valuable suggestions and comments, which helps a lot in improving the paper.

Open Access This article is distributed under the terms of the Creative Commons Attribution License which permits any use, distribution, and reproduction in any medium, provided the original author(s) and the source are credited.

\section{References}

1. Bergweiler, W.: Bloch's principle. Comput. Methods Funct. Theory 6, 77-108 (2006)

2. Chang, J.M.: Normality of meromorphic functions whose derivatives have 1-points. Arch. Math. 94, 555-564 (2010)

3. Chen, H.H., Gu, Y.X.: Improvement of Marty's criterion and its application. Sci. China Ser. A 36, 674-681 (1993)

4. Fang, M.L., Zalcman, L.: A note on normality and shared values. J. Aust. Math. Soc. 76, 141-150 (2004)

5. Gu, Y.X.: A criterion for normality of families of meromorphic functions (in Chinese). Sci. Sinica Special Issue 1 Math., 267-274 (1979)

6. Hayman, W.K.: Meromorphic Functions. Clarendon Press, Oxford (1964)

7. Hua, X.H.: On a problem of Hayman. Kodai Math. J. 13, 386-390 (1990)

8. Pang, X.C.: Bloch's principle and normal criterion. Sci. China Ser. A 32, 782-791 (1989)

9. Pang, X.C.: On normal criterion of meromorphic functions. Sci. China Ser. A 33, 521-527 (1990)

10. Pang, X.C., Zalcman, L.: Normal families and shared values. Bull. Lond. Math. Soc. 32, 325-331 (2000)

11. Schiff, J.: Normal Families. Springer, Berlin (1993)

12. Schwick, W.: Sharing values and normality. Arch Math. 38, 500-516 (1973)

13. Wang, Y.F., Fang, M.L.: Picard values and normal families of meromorphic functions with multiple zeros. Acta Math. Sinica (N.S.) 4, 17-26 (1998)

14. Yang, L.: Normality of families of meromorphic function. Sci. Sinica A 9, 898-908 (1986)

15. Yang, L.: Value Distribution Theory. Springer, Berlin (1993)

16. Zalcman, L.: A heuristic principle in complex function theory. Am. Math. Mon. 82, 813-817 (1975) 